\title{
Affordable Lab Kit for Controls Education
}

\section{Rebecca Marie Reck, University of Illinois, Urbana-Champaign}

Rebecca M. Reck just started Ph.D. studies in systems engineering at the University of Illinois at UrbanaChampaign. She completed her master's degree in electrical engineering at Iowa State University during her eight years at Rockwell Collins and her bachelor's degree in electrical engineering with a mathematics minor, from Rose-Hulman Institute of Technology in 2005. 


\section{Affordable Lab Kit for Controls Education}




\section{Affordable Lab Kit for Controls Education}

This research developed a modular, portable, and affordable laboratory kit and accompanying curriculum for two controls courses in the General Engineering Department. The objective is to design each kit to be assembled for under $\$ 100$ while replicating the educational functionality of a lab bench in a university controls laboratory. This will also allow older analog computers to be updated with newer technology that is more representative of what is currently used in industry ${ }^{1}$. This hardware kit will replace expensive equipment with an affordable alternative that can be easily shipped anywhere in the world and used by students with any computer. This greatly enhances the accessibility of the laboratory experience for students in budget-strapped campus laboratories and those participating in distance education or massive open online courses. The current laboratory equipment costs approximately $\$ 14,000$. Previous research shows that handson laboratory experiments help students understand and apply course material ${ }^{2}$.

Some affordable and transportable laboratory devices for engineering education have already been developed, such as the Mobile Studio IOBoard, which is centered on a custom-built board that "replicates the functionality of an oscilloscope, function generator, multimeter and power supplies" and is primarily used in introductory circuits courses ${ }^{3}$. This kit, like the IOBoard, will have the potential to enhance learning as well as the accessibility of the laboratory experience to students in budget-strapped campus laboratories and those in distance education.

The kit design initially consists of a Raspberry Pi (a fully functional ARM-based computer that is the size of a deck of cards), a DC motor, and the various components required for the GE320 (Introduction to Control Systems) lab exercises. Where possible, off-the-shelf components should be used to reduce the cost. Initial prototyping of the kit will be done with an Erector set, however parts will eventually be 3-D printed to simplify assembly. The cost to build this kit is approximately $\$ 114$.

In addition to the kit, each laboratory group will need a computer with MATLAB/Simulink and a network connection to the Raspberry Pi. The license for MATLAB and Simulink have not been included in the cost of either lab setup because it is assumed that a school with an existing controls curriculum will already have a site license.

A kit has been developed that will replace the technical functionality of four out of the six labs for GE320. It is currently about \$30 over the target, however with bulk ordering and diligent price comparisons the kit cost could be reduced even further. GE320 Lab 2 could not be replicated with this kit, at this time it is not clear if the educational objectives it to be included in the curriculum. This lab could be replaced or the one (or two) of the DC power supplies could be made available for students to use to complete the exercise. The impact of the use of this kit to student outcomes has not yet been assessed and is planned in future work.

1. H. Klee and J. Dumas, "Theory, simulation, experimentation: an integrated approach to teaching digital control systems,” Education, IEEE Transactions on, vol. 37, pp. 57-62, 1994.

2. K. A. Connor, B. Ferri and K. Meehan, "Models of mobile hands-on STEM education,” in 120th ASEE Annual Conference and Exposition, Atlanta, GA, 2013.

3. D. Millard, M. Chouikha and F. Berry, "Improving student intuition via Rensselaer's new mobile studio pedagogy,” in 114th Annual ASEE Conference and Exposition, 2007, Honolulu, HI, 2007. 